PROCEEDINGS OF THE

AMERICAN MATHEMATICAL SOCIETY

Volume 134, Number 3, Pages 897-908

S 0002-9939(05)08330-9

Article electronically published on September 28, 2005

\title{
BUBBLING PHENOMENA FOR FOURTH-ORDER FOUR-DIMENSIONAL PDES WITH EXPONENTIAL GROWTH
}

\author{
O. DRUET AND F. ROBERT
}

(Communicated by Jozef Dodziuk)

\begin{abstract}
We are concerned in this paper with the bubbling phenomenon for nonlinear fourth-order four-dimensional PDE's. The operators in the equations are perturbations of the bi-Laplacian. The nonlinearity is of exponential growth. Such equations arise naturally in statistical physics and geometry. As a consequence of our theorem we get a priori bounds for solutions of our equations.
\end{abstract}

We are concerned in this paper with understanding the bubbling phenomenon for fourth-order four-dimensional PDE's of exponential growth. Such equations arise naturally in statistical physics and in geometry (see [7] and 9]). In what follows, we let $(M, g)$ be a smooth compact Riemannian 4-manifold without boundary. We also let $\left(b_{\varepsilon}\right)_{\varepsilon>0}$ and $\left(f_{\varepsilon}\right)_{\varepsilon>0}$ be sequences of smooth functions on $M$, and we let $\left(A_{\varepsilon}\right)_{\varepsilon>0}$ be a sequence of smooth $(2,0)$-symetric tensor fields. We assume that $\left(b_{\varepsilon}\right),\left(f_{\varepsilon}\right)$ and $\left(A_{\varepsilon}\right)$ converge as $\varepsilon \rightarrow 0$ in the $C^{k}$-topologies, $k$ a positive integer, to limiting objects of the same nature, $b_{0}, f_{0}$ and $A_{0}$. Then we consider sequences $\left(u_{\varepsilon}\right)_{\varepsilon>0}$ of solutions of

$$
\Delta_{g}^{2} u_{\varepsilon}+R_{\varepsilon}\left(x, d u_{\varepsilon}\right)=f_{\varepsilon}(x) e^{u_{\varepsilon}},
$$

where $\Delta_{g}=-\operatorname{div}_{g}(\nabla$.$) is the Laplace-Beltrami operator and$

$$
R_{\varepsilon}(x, d u)=-\operatorname{div}_{g}\left(A_{\varepsilon} d u\right)+b_{\varepsilon} .
$$

Following standard terminology, we say that the $u_{\varepsilon}$ 's blow up if $u_{\varepsilon}\left(x_{\varepsilon}\right) \rightarrow+\infty$ as $\varepsilon \rightarrow 0$ for a sequence $\left(x_{\varepsilon}\right)$ of points in $M$. We let

$$
L_{0}=\Delta_{g}^{2} u-\operatorname{div}_{g}\left(A_{0} d u\right)
$$

be the limit operator in (11). At last, we let $G$ be the Green function of $L_{0}$. The Green function is unique up to a constant when the kernel of $L_{0}$ consists only of constants. We write $G$ as

$$
G(x, y)=\frac{1}{8 \pi^{2}} \ln \frac{1}{d_{g}(x, y)}+\beta(x, y)
$$

Received by the editors September 29, 2004.

2000 Mathematics Subject Classification. Primary 58E30, 58J05, 35J35.

Key words and phrases. Concentration estimates, fourth-order equations, compactness.

(C)2005 American Mathematical Society Reverts to public domain 28 years from publication 
for $(x, y) \in M \times M \backslash D$, with $D=\{(x, x), x \in M\}$ the diagonal in $M \times M$, where $\beta \in C^{1}(M \times M)$. We let $\varphi$ be the function given by

$$
\varphi(x)=\int_{M} G(x, y) b_{0}(y) d v_{g}(y) .
$$

For $u$ a function on $M$ we let

$$
\bar{u}=\frac{1}{\operatorname{Vol}_{g}(M)} \int_{M} u d v_{g}
$$

be the mean value of $u$, where $\operatorname{Vol}_{g}(M)$ is the volume of $M$ with respect to $g$. Our theorem is stated as follows:

Theorem 1. Let $(M, g)$ be a smooth compact Riemannian manifold of dimension 4 without boundary. Let $\left(u_{\varepsilon}\right)$ be a blowing-up sequence of solutions of (11). Assume that the kernel of $L_{0}$ consists only of constants and that $f_{0}$ is a positive function on $M$. Then

$$
\int_{M} b_{0} d v_{g}=64 \pi^{2} N
$$

for some $N \in \mathbb{N}^{\star}$. Moreover there exists a finite subset $S \subset M$, consisting of $N$ points $x_{i}, i=1, \ldots, N$, such that

$$
u_{\varepsilon}-\bar{u}_{\varepsilon} \rightarrow 64 \pi^{2} \sum_{i=1}^{N} G\left(x_{i}, .\right)-\varphi
$$

in $C_{\text {loc }}^{4}(M \backslash S)$. At last, we have that

$$
64 \pi^{2} \nabla_{y} \beta\left(x_{i}, x_{i}\right)+64 \pi^{2} \sum_{j \neq i} \nabla_{x} G\left(x_{i}, x_{j}\right)-\nabla \varphi\left(x_{i}\right)=-\frac{\nabla f_{0}\left(x_{i}\right)}{f_{0}\left(x_{i}\right)}
$$

for all $i=1, \ldots, N$.

The proof of Theorem 1 comes with strong pointwise estimates on the $u_{\varepsilon}$ 's and the observation that concentration points are isolated (we refer to section 1 for details). This should be compared to the more intricate situation of Yamabe-type equations for which concentration points are not necessarily isolated (see [3, 4, 5, 6]). Independently, as is easily checked, a priori $C^{4}$-bounds on sequences of solutions follow from the above theorem when $\int_{M} b_{0} d v_{g} \notin 64 \pi^{2} \mathbb{N}$. This includes compactness of the geometric Paneitz equation with arbitrary prescribed $Q$-curvature (we refer to the nice surveys [1] and [2] for material on the $Q$-curvature). Such a priori $C^{4}$ bounds should be regarded as a first step towards a Morse theory for the equations we consider in this paper. We refer to [11], where this question was handled in the case of the Yamabe equation.

\section{Proof of Theorem 1}

Let us assume that we have a sequence $\left(u_{\varepsilon}\right)$ of smooth solutions of

$$
L_{\varepsilon} u_{\varepsilon}+b_{\varepsilon}(x)=f_{\varepsilon}(x) e^{u_{\varepsilon}},
$$

where $L_{\varepsilon}=\Delta_{g}^{2}-\operatorname{div}_{g}\left(A_{\varepsilon} d\right.$. ). Since we assumed that $\operatorname{Ker} L_{0}=\{$ constants $\}$, it is clear that $\operatorname{Ker} L_{\varepsilon}=\{$ constants $\}$ for all $\varepsilon>0$ small enough. Thus, if the sequence $\left(u_{\varepsilon}\right)$ is bounded from above, it follows from standard elliptic theory that $\left(u_{\varepsilon}\right)$ is 
uniformly bounded in $C^{4}(M)$ except if $\int_{M} b_{0} d v_{g}=0$. This clarifies the remarks after the theorem. From now on, we assume that the $u_{\varepsilon}$ 's blow up, i.e. that

$$
\max _{M} u_{\varepsilon} \rightarrow+\infty \text { as } \varepsilon \rightarrow 0 .
$$

Before starting the proof of Theorem 1, we note that, integrating equation (4),

$$
\int_{M} f_{\varepsilon} e^{u_{\varepsilon}} d v_{g}=\int_{M} b_{\varepsilon} d v_{g}=\int_{M} b_{0} d v_{g}+o(1) .
$$

We divide the proof into several steps. The first step is as follows:

STEP 1 - Assume that (5) holds. Then there exist $N \in \mathbb{N}^{\star}$ and $N$ sequences $\left(x_{i, \varepsilon}\right)$ of converging points in $M$ such that, after passing to a subsequence, the following assertions hold:

a) $\frac{d_{g}\left(x_{i, \varepsilon}, x_{j, \varepsilon}\right)}{\mu_{i, \varepsilon}} \rightarrow+\infty$ as $\varepsilon \rightarrow 0$ for all $i, j=1, \ldots, N, i \neq j$, where

$$
f_{\varepsilon}\left(x_{i, \varepsilon}\right) \mu_{i, \varepsilon}^{4} e^{u_{\varepsilon}\left(x_{i, \varepsilon}\right)}=1
$$

b) We have that

$$
v_{i, \varepsilon}(x)=u_{\varepsilon}\left(\exp _{x_{i, \varepsilon}}\left(\mu_{i, \varepsilon} x\right)\right)-u_{\varepsilon}\left(x_{i, \varepsilon}\right) \rightarrow V_{0}(x)=-4 \ln \left(1+\frac{|x|^{2}}{8 \sqrt{6}}\right)
$$

in $C_{\text {loc }}^{4}\left(\mathbb{R}^{4}\right)$ as $\varepsilon \rightarrow 0$ for all $i=1, \ldots, N$.

c) For all $i=1, \ldots, N$, we have that

$$
\lim _{R \rightarrow+\infty} \lim _{\varepsilon \rightarrow 0} \int_{B_{x_{i, \varepsilon}}\left(R \mu_{i, \varepsilon}\right)} f_{\varepsilon} e^{u_{\varepsilon}} d v_{g}=64 \pi^{2} .
$$

d) At last, there exists $C>0$ such that

$$
\left(\inf _{i=1, \ldots, N} d_{g}\left(x_{i, \varepsilon}, x\right)^{4}\right) e^{u_{\varepsilon}(x)} \leq C
$$

for all $\varepsilon>0$ and all $x \in M$.

Proof of Step 1. We briefly sketch the proof below and we refer to 10, for the details. We let $x_{\varepsilon} \in M$ be such that $u_{\varepsilon}\left(x_{\varepsilon}\right)=\max _{M} u_{\varepsilon}$. By (5),$u_{\varepsilon}\left(x_{\varepsilon}\right) \rightarrow+\infty$ as $\varepsilon \rightarrow 0$. We let $\mu_{\varepsilon}>0$ be defined by

$$
f_{\varepsilon}\left(x_{\varepsilon}\right) \mu_{\varepsilon}^{4} e^{u_{\varepsilon}\left(x_{\varepsilon}\right)}=1
$$

so that $\mu_{\varepsilon} \rightarrow 0$ as $\varepsilon \rightarrow 0$. For $x \in B_{0}\left(\delta \mu_{\varepsilon}^{-1}\right)$, the Euclidean ball of center 0 and radius $\delta \mu_{\varepsilon}^{-1}, \delta>0$ small fixed, we let

$$
\begin{aligned}
& v_{\varepsilon}(x)=u_{\varepsilon}\left(\exp _{x_{\varepsilon}}\left(\mu_{\varepsilon} x\right)\right)-u_{\varepsilon}\left(x_{\varepsilon}\right), \\
& g_{\varepsilon}(x)=\left(\exp _{x_{\varepsilon}}^{\star} g\right)\left(\mu_{\varepsilon} x\right), \tilde{A}_{\varepsilon}(x)=\left(\exp _{x_{\varepsilon}}^{\star} A_{\varepsilon}\right)\left(\mu_{\varepsilon} x\right), \\
& \tilde{b}_{\varepsilon}(x)=b_{\varepsilon}\left(\exp _{x_{\varepsilon}}\left(\mu_{\varepsilon} x\right)\right) \text { and } \tilde{f}_{\varepsilon}(x)=f_{\varepsilon}\left(\exp _{x_{\varepsilon}}\left(\mu_{\varepsilon} x\right)\right) .
\end{aligned}
$$

We then have that

$$
\Delta_{g_{\varepsilon}}^{2} v_{\varepsilon}-\mu_{\varepsilon}^{2} d i v_{g_{\varepsilon}}\left(\tilde{A}_{\varepsilon} d v_{\varepsilon}\right)+\mu_{\varepsilon}^{4} \tilde{b}_{\varepsilon}=\frac{\tilde{f}_{\varepsilon}}{f_{\varepsilon}\left(x_{\varepsilon}\right)} e^{v_{\varepsilon}}
$$

in $B_{0}\left(\delta \mu_{\varepsilon}^{-1}\right)$. We write with the Green representation formula that

$$
u_{\varepsilon}(x)-\bar{u}_{\varepsilon}=\int_{M} G_{\varepsilon}(x, y) L_{\varepsilon} u_{\varepsilon}(y) d v_{g}(y)
$$


for all $x \in M$, where $G_{\varepsilon}$ is the Green function of $L_{\varepsilon}$. Using equation (4) and differentiating the above with respect to $x$, we obtain for $k=1,2,3$ that

$$
\begin{aligned}
\left|\nabla^{k} u_{\varepsilon}\right|_{g}(x) & \leq \int_{M}\left|\nabla_{x}^{k} G_{\varepsilon}(x, y)\right|_{g}\left|f_{\varepsilon}(y) e^{u_{\varepsilon}(y)}-b_{\varepsilon}(y)\right| d v_{g}(y) \\
& \leq \int_{M}\left|\nabla_{x}^{k} G_{\varepsilon}(x, y)\right|_{g} f_{\varepsilon}(y) e^{u_{\varepsilon}(y)} d v_{g}(y)+O(1)
\end{aligned}
$$

since $b_{\varepsilon} \rightarrow b_{0}$ in $C^{0}(M)$ as $\varepsilon \rightarrow 0$. Let $y_{\varepsilon} \in B_{x_{\varepsilon}}\left(R \mu_{\varepsilon}\right), R>0$ fixed. We write that

$$
\begin{aligned}
& \int_{M}\left|\nabla_{x}^{k} G\left(y_{\varepsilon}, y\right)\right|_{g} e^{u_{\varepsilon}(y)} d v_{g}(y) \\
& =O\left(\mu_{\varepsilon}^{-k} \int_{M \backslash B_{y_{\varepsilon}}\left(\mu_{\varepsilon}\right)} e^{u_{\varepsilon}} d v_{g}\right)+O\left(e^{u_{\varepsilon}\left(x_{\varepsilon}\right)} \int_{B_{y_{\varepsilon}}\left(\mu_{\varepsilon}\right)} d_{g}\left(y_{\varepsilon}, y\right)^{-k} d v_{g}(y)\right) \\
& =O\left(\mu_{\varepsilon}^{-k}\right)
\end{aligned}
$$

thanks to the fact that $u_{\varepsilon} \leq u_{\varepsilon}\left(x_{\varepsilon}\right)$, to (7) and to standard estimates on the Green function (which are uniform in $\varepsilon$ ). Together with the definition (8) of $v_{\varepsilon}$, this gives that $\left(v_{\varepsilon}\right)$ is uniformly bounded in $C^{3}(K)$ for all compact subsets $K$ of $\mathbb{R}^{4}$. Standard elliptic theory then gives thanks to equation (9) that

$$
\lim _{\varepsilon \rightarrow 0} v_{\varepsilon}=V_{0} \text { in } C_{l o c}^{4}\left(\mathbb{R}^{4}\right)
$$

where $V_{0}$ is a solution of

$$
\Delta_{\xi}^{2} V_{0}=e^{V_{0}}
$$

in $\mathbb{R}^{4}$ satisfying $V_{0}(x) \leq V_{0}(0)=0$ for all $x \in \mathbb{R}^{4}$. Moreover, since

$$
\lim _{\varepsilon \rightarrow 0} \int_{B_{x_{\varepsilon}}\left(R \mu_{\varepsilon}\right)} f_{\varepsilon} e^{u_{\varepsilon}} d v_{g}=\int_{B_{0}(R)} e^{V_{0}} d x,
$$

equation (6) implies that $e^{V_{0}} \in L^{1}\left(\mathbb{R}^{4}\right)$. From the classification of the solutions of equation (11) by Lin [8, we get that either

$$
V_{0}(x)=-4 \ln \left(1+\frac{|x|^{2}}{8 \sqrt{6}}\right)
$$

or there exists $a>0$ such that

$$
\Delta_{\xi} V_{0} \geq a
$$

in $\mathbb{R}^{4}$. Let us prove that we are in the first situation. For that purpose, we write with the Green representation formula and equation (4) that

$$
\begin{aligned}
& \int_{B_{0}(R)}\left|\Delta_{g_{\varepsilon}} v_{\varepsilon}\right|_{g_{\varepsilon}} d v_{g_{\varepsilon}}=\mu_{\varepsilon}^{-2} \int_{B_{x_{\varepsilon}}\left(R \mu_{\varepsilon}\right)}\left|\Delta_{g} u_{\varepsilon}\right|_{g} d v_{g} \\
& \leq C \mu_{\varepsilon}^{-2} \int_{x \in B_{x_{\varepsilon}}\left(R \mu_{\varepsilon}\right)} \int_{y \in M}\left|\Delta_{g, x} G_{\varepsilon}(x, y)\right|_{g}\left(e^{u_{\varepsilon}(y)}+1\right) d v_{g}(y) d v_{g}(x) \\
& \leq C \mu_{\varepsilon}^{-2} \int_{y \in M}\left(e^{u_{\varepsilon}(y)}+1\right)\left(\int_{x \in B_{x_{\varepsilon}}\left(R \mu_{\varepsilon}\right)} d_{g}(x, y)^{-2} d v_{g}(x)\right) d v_{g}(y) \\
& \leq C R^{2}
\end{aligned}
$$


thanks to standard estimates on the Green function and to ([6), where $C>0$ denotes some constant independent of $R$ and $\varepsilon>0$. Letting $\varepsilon \rightarrow 0$, we get that

$$
\int_{B_{0}(R)}\left|\Delta_{\xi} V_{0}\right|_{\xi} d x \leq C R^{2}
$$

for all $R>0$. This clearly eliminates the possibility (13). Then (12) must hold. It is then easily checked that

$$
\lim _{R \rightarrow+\infty} \lim _{\varepsilon \rightarrow 0} \int_{B_{x_{\varepsilon}}\left(R \mu_{\varepsilon}\right)} f_{\varepsilon} e^{u_{\varepsilon}} d v_{g}=\int_{\mathbb{R}^{4}} e^{V_{0}} d x=64 \pi^{2} .
$$

For $k \geq 1$, we say that $\mathcal{H}_{k}$ holds if there exist $\left(x_{i, \varepsilon}\right)_{i=1, \ldots, k} k$ converging sequences of points in $M$ and $\left(\mu_{i, \varepsilon}\right)_{i=1, \ldots, k} k$ sequences of positive real numbers going to 0 as $\varepsilon \rightarrow 0$ such that $f_{\varepsilon}\left(x_{i, \varepsilon}\right) \mu_{i, \varepsilon}^{4} e^{u_{\varepsilon}\left(x_{i, \varepsilon}\right)}=1$ and such that, after passing to a subsequence, the following assertions hold:

$\left(A_{k}^{1}\right) \frac{d_{g}\left(x_{i, \varepsilon}, x_{j, \varepsilon}\right)}{\mu_{i, \varepsilon}} \rightarrow+\infty$ as $\varepsilon \rightarrow 0$ for all $i, j=1, \ldots, N, i \neq j$.

$\left(A_{k}^{2}\right)$ We have that

$$
v_{i, \varepsilon}(x)=u_{\varepsilon}\left(\exp _{x_{i, \varepsilon}}\left(\mu_{i, \varepsilon} x\right)\right)-u_{\varepsilon}\left(x_{i, \varepsilon}\right) \rightarrow V_{0}(x)=-4 \ln \left(1+\frac{|x|^{2}}{8 \sqrt{6}}\right)
$$

in $C_{l o c}^{4}\left(\mathbb{R}^{4}\right)$ as $\varepsilon \rightarrow 0$ for all $i=1, \ldots, N$.

$\left(A_{k}^{3}\right)$ For all $i=1, \ldots, N$, we have that

$$
\lim _{R \rightarrow+\infty} \lim _{\varepsilon \rightarrow 0} \int_{B_{x_{i, \varepsilon}}\left(R \mu_{i, \varepsilon}\right)} f_{\varepsilon} e^{u_{\varepsilon}} d v_{g}=64 \pi^{2} .
$$

Clearly, with what we said above, $\mathcal{H}_{1}$ holds. Now we let $k \geq 1$ and assume that $\mathcal{H}_{k}$ holds. We also assume that

$$
\sup _{M} R_{k, \varepsilon}(x)^{4} e^{u_{\varepsilon}(x)} \rightarrow+\infty \text { as } \varepsilon \rightarrow 0,
$$

where

$$
R_{k, \varepsilon}(x)=\min _{i=1, \ldots, k} d_{g}\left(x_{i, \varepsilon}, x\right) .
$$

We prove in the following that, in this situation, $\mathcal{H}_{k+1}$ holds. For that purpose, we let $x_{k+1, \varepsilon} \in M$ be such that

$$
R_{k, \varepsilon}\left(x_{k+1, \varepsilon}\right)^{4} e^{u_{\varepsilon}\left(x_{k+1, \varepsilon}\right)}=\sup _{M} R_{k, \varepsilon}(x)^{4} e^{u_{\varepsilon}(x)}
$$

and we set

$$
\mu_{k+1, \varepsilon}=\left(\frac{1}{f_{\varepsilon}\left(x_{k+1, \varepsilon}\right) e^{u_{\varepsilon}\left(x_{k+1, \varepsilon}\right)}}\right)^{\frac{1}{4}} .
$$

Since $M$ is compact, (15) implies that $\mu_{k+1, \varepsilon} \rightarrow 0$ as $\varepsilon \rightarrow 0$ and that

$$
\frac{d_{g}\left(x_{i, \varepsilon}, x_{k+1, \varepsilon}\right)}{\mu_{k+1, \varepsilon}} \rightarrow+\infty \text { as } \varepsilon \rightarrow 0
$$

for all $i=1, \ldots, k$. Thanks to $\left(A_{k}^{2}\right)$, it is also easily checked that $\frac{d_{g}\left(x_{i, \varepsilon}, x_{k+1, \varepsilon}\right)}{\mu_{i, \varepsilon}} \rightarrow$ $+\infty$ as $\varepsilon \rightarrow 0$ for all $i=1, \ldots, k$ so that $\left(A_{k+1}^{1}\right)$ holds. It follows from (16) and (17) that

$$
\lim _{\varepsilon \rightarrow 0} \sup _{z \in B_{x_{k+1, \varepsilon}}\left(R \mu_{k+1, \varepsilon}\right)}\left(u_{\varepsilon}(z)-u_{\varepsilon}\left(x_{k+1, \varepsilon}\right)\right)=0 .
$$


Mimicking what we did above thanks to the Green representation formula, one then proves that, after passing to a subsequence,

$$
u_{\varepsilon}\left(\exp _{x_{k+1, \varepsilon}}\left(\mu_{k+1, \varepsilon} x\right)\right)-u_{\varepsilon}\left(x_{k+1, \varepsilon}\right) \rightarrow V_{0}(x)
$$

in $C_{\text {loc }}^{4}\left(\mathbb{R}^{4}\right)$ as $\varepsilon \rightarrow 0$. And, as a consequence,

$$
\lim _{R \rightarrow+\infty} \lim _{\varepsilon \rightarrow 0} \int_{B_{x_{k+1, \varepsilon}}\left(R \mu_{k+1, \varepsilon}\right)} f_{\varepsilon} e^{u_{\varepsilon}} d v_{g}=64 \pi^{2} .
$$

Recollecting the information above, one gets that $\mathcal{H}_{k+1}$ holds. Since $\left(A_{k}^{1}\right)$ and $\left(A_{k}^{3}\right)$ of $\mathcal{H}_{k}$ imply that

$$
\int_{M} f_{\varepsilon} e^{u_{\varepsilon}} d v_{g} \geq 64 \pi^{2} k+o(1),
$$

we easily get, thanks to (6), that there exists a maximal $k, 1 \leq k \leq \frac{1}{64 \pi^{2}} \int_{M} b_{0} d v_{g}$, such that $\mathcal{H}_{k}$ holds. Arriving to this maximal $k$, we get that (15) cannot hold. Writing $k=N$, we have finished the proof of Step 1 .

SteP 2 - For $k=1,2,3$, there exists $C_{k}>0$ such that

$$
R_{\varepsilon}(x)^{k}\left|\nabla^{k} u_{\varepsilon}\right|_{g}(x) \leq C_{k}
$$

for all $x \in M$ and all $\varepsilon>0$. Here,

$$
R_{\varepsilon}(x)=\inf _{i=1, \ldots, N} d_{g}\left(x_{i, \varepsilon}, x\right)
$$

where the $x_{i, \varepsilon}$ 's are as in Step 1.

Proof of Step 2. We use again the Green representation for $u_{\varepsilon}$ that we differentiate. We let $x_{\varepsilon} \in M$ be such that $x_{\varepsilon} \neq x_{i, \varepsilon}$ for all $i=1, \ldots, N$. Note that, for $x_{\varepsilon}=x_{i, \varepsilon}$, the estimates of the proposition are obvious. We write, thanks to standard estimates on the Green function, that

$$
\left|\nabla^{k} u_{\varepsilon}\right|_{g}\left(x_{\varepsilon}\right)=O\left(\int_{M} \frac{1}{d_{g}\left(x_{\varepsilon}, y\right)^{k}} e^{u_{\varepsilon}(y)} d v_{g}(y)\right)+O(1) .
$$

For $i=1, \ldots, N$, we let

$$
\Omega_{i, \varepsilon}=\left\{y \in M, R_{\varepsilon}(y)=d_{g}\left(x_{i, \varepsilon}, y\right)\right\}
$$

and we write that

$$
\begin{aligned}
& \int_{\Omega_{i, \varepsilon}} \frac{1}{d_{g}\left(x_{\varepsilon}, y\right)^{k}} e^{u_{\varepsilon}(y)} d v_{g}(y) \\
& =O\left(\frac{1}{d_{g}\left(x_{\varepsilon}, x_{i, \varepsilon}\right)^{k}} \int_{\Omega_{i, \varepsilon} \cap B_{x_{i, \varepsilon}}\left(\frac{d_{g}\left(x_{\varepsilon}, x_{i, \varepsilon}\right)}{2}\right)} e^{u_{\varepsilon}} d v_{g}\right) \\
& +O\left(\int_{\Omega_{i, \varepsilon} \backslash B_{x_{i, \varepsilon}}\left(\frac{d_{g}\left(x_{i, \varepsilon}, x_{\varepsilon}\right)}{2}\right)} \frac{1}{d_{g}\left(x_{\varepsilon}, y\right)^{k}} \frac{1}{d_{g}\left(y, x_{i, \varepsilon}\right)^{4}} d v_{g}(y)\right) \\
& =O\left(\frac{1}{d_{g}\left(x_{\varepsilon}, x_{i, \varepsilon}\right)^{k}}\right)
\end{aligned}
$$

thanks to assertion d) of Step 1, to (6) and to some straightforward computations. Step 2 clearly follows. 
STEP 3 - For any $1 \leq \nu<2$, there exists $\delta_{\nu}>0$ and $C_{\nu}>0$ such that

$$
\mu_{i, \varepsilon}^{4(1-\nu)} d_{g}\left(x_{i, \varepsilon}, x\right)^{4 \nu} e^{u_{\varepsilon}(x)} \leq C_{\nu}
$$

for all $i=1, \ldots, N$, all $\varepsilon>0$ and all $x \in B_{x_{i, \varepsilon}}\left(\delta_{\nu}\right)$ where $x_{i, \varepsilon}$ and $\mu_{i, \varepsilon}$ are as in Step 1. In particular, we have that

$$
d_{g}\left(x_{i, \varepsilon}, x_{j, \varepsilon}\right) \geq \delta_{0}
$$

for all $i, j \in\{1, \ldots, N\}, i \neq j$, where $\delta_{0}>0$ is independent of $\varepsilon$ and $i, j$. At last, this implies that $\bar{u}_{\varepsilon} \rightarrow-\infty$ as $\varepsilon \rightarrow 0$.

Proof of Step 3. Fix $1 \leq \nu<2$. We set for $i=1, \ldots, N$

$$
R_{i, \varepsilon}=\min _{j \neq i} d_{g}\left(x_{i, \varepsilon}, x_{j, \varepsilon}\right)
$$

and we take some $i \in\{1, \ldots, N\}$ such that there exists $\theta>0$ such that

$$
R_{i, \varepsilon} \leq \theta R_{j, \varepsilon}
$$

for all $j \in\{1, \ldots, N\}$. We set

$$
\varphi_{i, \varepsilon}(r)=r^{4 \nu} \exp \left(\left(\operatorname{Vol}_{g}\left(\partial B_{x_{i, \varepsilon}}(r)\right)\right)^{-1} \int_{\partial B_{x_{i, \varepsilon}}(r)} u_{\varepsilon} d \sigma_{g}\right)
$$

for $0 \leq r<i n j_{g}(M)$. A simple consequence of assertion b) of Step 1 is that

$$
\varphi_{i, \varepsilon}^{\prime}\left(R \mu_{i, \varepsilon}\right)<0
$$

for $\varepsilon>0$ small and all $R \geq R_{\nu}$ where $R_{\nu}^{2}=\frac{16 \sqrt{6} \nu}{2-\nu}$. We define $r_{i, \varepsilon}$ by

$$
r_{i, \varepsilon}=\inf \left\{R_{\nu} \mu_{i, \varepsilon} \leq r \leq \frac{R_{i, \varepsilon}}{2} \text { s.t. } \varphi_{i, \varepsilon}^{\prime}(r)<0 \text { in }\left[R_{\nu} \mu_{i, \varepsilon}, r\right)\right\} .
$$

Note that, by (21), we have that

$$
\frac{r_{i, \varepsilon}}{\mu_{i, \varepsilon}} \rightarrow+\infty \text { as } \varepsilon \rightarrow 0
$$

Let us assume that

$$
r_{i, \varepsilon} \rightarrow 0 \text { as } \varepsilon \rightarrow 0
$$

For $x \in B_{0}\left(\delta r_{i, \varepsilon}^{-1}\right), \delta>0$ small fixed, we set

$$
v_{i, \varepsilon}(x)=u_{\varepsilon}\left(\exp _{x_{i, \varepsilon}}\left(r_{i, \varepsilon} x\right)\right)-C_{i, \varepsilon},
$$

where

$$
C_{i, \varepsilon}=\left(\operatorname{Vol}_{g}\left(\partial B_{x_{i, \varepsilon}}\left(r_{i, \varepsilon}\right)\right)\right)^{-1} \int_{\partial B_{x_{i, \varepsilon}}\left(r_{i, \varepsilon}\right)} u_{\varepsilon} d \sigma_{g} .
$$

We also set, for $j \in \mathcal{S}_{i}=\left\{j \neq i\right.$ s.t. $\left.d_{g}\left(x_{i, \varepsilon}, x_{j, \varepsilon}\right)=O\left(r_{i, \varepsilon}\right)\right\}$,

$$
\tilde{x}_{j, \varepsilon}=r_{i, \varepsilon}^{-1} \exp _{x_{i, \varepsilon}}^{-1}\left(x_{j, \varepsilon}\right) \text { and } \tilde{x}_{j}=\lim _{\varepsilon \rightarrow 0} \tilde{x}_{j, \varepsilon}
$$

after passing to a subsequence, if necessary. Note that, thanks to (18), to (22) and to the choice of $i$ we made (see (19)), we have that $\left|\tilde{x}_{j}\right| \geq 2$ for all $j \in \mathcal{S}_{i}$ and that $\left|\tilde{x}_{j}-\tilde{x}_{k}\right| \geq \frac{2}{\theta}$ for all $j, k \in \mathcal{S}_{i}, j \neq k$. By equation (4), we have that

$$
\Delta_{g_{i, \varepsilon}}^{2} v_{i, \varepsilon}-r_{i, \varepsilon}^{2} d i v_{g_{i, \varepsilon}}\left(A_{i, \varepsilon} \nabla v_{i, \varepsilon}\right)+r_{i, \varepsilon}^{4} b_{i, \varepsilon}=f_{i, \varepsilon} \varphi_{i, \varepsilon}\left(r_{i, \varepsilon}\right) r_{i, \varepsilon}^{4(1-\nu)} e^{v_{i, \varepsilon}}
$$


in $B_{0}\left(\delta r_{i, \varepsilon}^{-1}\right)$, where

$$
\begin{aligned}
& g_{i, \varepsilon}(x)=\left(\exp _{x_{i, \varepsilon}}^{\star} g\right)\left(r_{i, \varepsilon} x\right), A_{i, \varepsilon}(x)=\left(\exp _{x_{i, \varepsilon}}^{\star} A_{\varepsilon}\right)\left(r_{i, \varepsilon} x\right), \\
& b_{i, \varepsilon}(x)=b_{\varepsilon}\left(\exp _{x_{i, \varepsilon}}\left(r_{i, \varepsilon} x\right)\right) \text { and } f_{i, \varepsilon}(x)=f_{\varepsilon}\left(\exp _{x_{i, \varepsilon}}\left(r_{i, \varepsilon} x\right)\right) .
\end{aligned}
$$

Thanks to Step 2, we know that $\left(v_{i, \varepsilon}\right)$ is uniformly bounded in $C^{3}(K)$ for all compact subsets $K$ of $\mathbb{R}^{4} \backslash\left\{0, \tilde{x}_{j}\right\}_{j \in \mathcal{S}_{i}}$. Thanks to the definition (22) of $r_{i, \varepsilon}$ and to (23), we have that

$$
\varphi_{i, \varepsilon}\left(r_{i, \varepsilon}\right) \leq \varphi_{i, \varepsilon}\left(R \mu_{i, \varepsilon}\right)
$$

for all $R>R_{\nu}$. Thanks to assertion b) of Step 1 and to (23), it is now rather easily checked that

$$
\lim _{R \rightarrow+\infty} \lim _{\varepsilon \rightarrow 0} \varphi_{i, \varepsilon}\left(R \mu_{i, \varepsilon}\right) r_{i, \varepsilon}^{4(1-\nu)}=0
$$

since $1 \leq \nu<2$. Thus standard elliptic theory leads, thanks to (28) and (29), that, after passing to a subsequence,

$$
v_{i, \varepsilon} \rightarrow H_{i} \text { in } C_{l o c}^{4}\left(\mathbb{R}^{4} \backslash\left\{0, \tilde{x}_{j}\right\}_{j \in \mathcal{S}_{i}}\right) \text { as } \varepsilon \rightarrow 0,
$$

where $H_{i}$ satisfies

$$
\Delta_{\xi}^{2} H_{i}=0 \text { in } \mathbb{R}^{4} \backslash\left\{0, \tilde{x}_{j}\right\}_{j \in \mathcal{S}_{i}} .
$$

Moreover, thanks to Step 2, we have that, for $l=1,2,3$,

$$
R(x)^{l}\left|\nabla^{l} H_{i}(x)\right|_{\xi} \leq C_{l} \text { in } \mathbb{R}^{4} \backslash\left\{0, \tilde{x}_{j}\right\}_{j \in \mathcal{S}_{i}},
$$

where

$$
R(x)=\min \left\{|x| ;\left|x-\tilde{x}_{j}\right|\right\}_{j \in \mathcal{S}_{i}} .
$$

Equation (32) easily allows us to prove that

$$
H_{i}(x)=\alpha \ln \frac{1}{|x|}+\sum_{j \in \mathcal{S}_{i}} \alpha_{j} \ln \frac{1}{\left|x-\tilde{x}_{j}\right|}+\beta,
$$

where $\alpha, \beta$ and the $\alpha_{j}$ 's are real numbers. Integrating equation (28) over $B_{0}(1)$ and passing to the limit as $\varepsilon \rightarrow 0$ thanks to (29), (30) and (33), we obtain that

$$
\lim _{\varepsilon \rightarrow 0} \varphi_{i, \varepsilon}\left(r_{i, \varepsilon}\right) r_{i, \varepsilon}^{4(1-\nu)} \int_{B_{0}(1)} f_{i, \varepsilon} e^{v_{i, \varepsilon}} d v_{g_{i, \varepsilon}}=-\int_{\partial B_{0}(1)} \partial_{\nu} \Delta_{\xi} H_{i} d \sigma_{\xi}=8 \alpha \pi^{2} .
$$

With a change of variable, we get that

$$
\varphi_{i, \varepsilon}\left(r_{i, \varepsilon}\right) r_{i, \varepsilon}^{4(1-\nu)} \int_{B_{0}(1)} f_{i, \varepsilon} e^{v_{i, \varepsilon}} d v_{g_{i, \varepsilon}}=\int_{B_{x_{i, \varepsilon}}\left(r_{i, \varepsilon}\right)} f_{\varepsilon} e^{u_{\varepsilon}} d v_{g}
$$

so that

$$
\lim _{\varepsilon \rightarrow 0} \int_{B_{x_{i, \varepsilon}}\left(r_{i, \varepsilon}\right)} f_{\varepsilon} e^{u_{\varepsilon}} d v_{g}=8 \alpha \pi^{2} .
$$

Step 2 with $k=1$ together with the definitions of $R_{i, \varepsilon}$ and $r_{i, \varepsilon}$ gives the existence of some $C>0$ such that for any $0 \leq r \leq 3 / 2$,

$$
\left|u_{\varepsilon}\left(\exp _{x_{i, \varepsilon}}\left(r_{i, \varepsilon} x\right)\right)-u_{\varepsilon}\left(\exp _{x_{i, \varepsilon}}\left(r_{i, \varepsilon} y\right)\right)\right| \leq C
$$


for all $x, y \in \mathbb{R}^{4}$ such that $|x|=|y|=r$. With point b) of Step 1, (22) and (23), we then get that for any $\eta>0$, there exists $R_{\eta}>0$ such that for any $R>R_{\eta}$, we have that

$$
d_{g}\left(x, x_{i, \varepsilon}\right)^{4 \nu} e^{u_{\varepsilon}(x)} \leq \eta \mu_{i, \varepsilon}^{4(\nu-1)}
$$

for all $x \in B_{x_{i, \varepsilon}}\left(r_{i, \varepsilon}\right) \backslash B_{x_{i, \varepsilon}}\left(R \mu_{i, \varepsilon}\right)$. With point b) of Step 1 and (35), we get that

$$
\lim _{\varepsilon \rightarrow 0} \int_{B_{x_{i, \varepsilon}}\left(r_{i, \varepsilon}\right)} f_{\varepsilon} e^{u_{\varepsilon}} d v_{g}=64 \pi^{2} .
$$

With (34), we obtain that $\alpha=8$. Integrating on $B_{\tilde{x}_{j}}(\delta)$ for $\delta>0$ small instead of $B_{0}(1)$, one proves in the same way that $\alpha_{j} \geq 8$ for all $j \in \mathcal{S}_{i}$. We let

$$
\bar{H}_{i}(r)=\frac{1}{2 \pi^{2} r^{3}} \int_{\partial B_{0}(r)} H_{i}(x) d \sigma .
$$

A simple computation gives

$$
\frac{d}{d r}\left(r^{4 \nu} e^{\bar{H}_{i}(r)}\right)=4\left(\nu-2-\left(\sum_{j \in \mathcal{S}_{i}} \frac{\alpha_{j}}{8\left|\tilde{x}_{j}\right|^{2}}\right) r^{2}\right) r^{4 \nu-1} e^{\bar{H}_{i}(r)}
$$

for $r \in\left(0, \frac{3}{2}\right)$. Since $\nu<2$, we get in particular that

$$
\frac{d}{d r}\left(r^{4 \nu} e^{\bar{H}_{i}(r)}\right)(1)<0 .
$$

This clearly proves that

$$
r_{i, \varepsilon}=\frac{R_{i, \varepsilon}}{2}
$$

for all $i$ such that (19) holds. Thanks to (24), this in turn implies that $R_{i, \varepsilon} \rightarrow 0$ and that $\mathcal{S}_{j} \neq \emptyset$. Note that, for the moment, we have proved, with the help of Step 2 (see (35)), that the estimate of Step 3 holds if for any $i \in\{1, \ldots, N\}$, we have that $R_{i, \varepsilon} \nrightarrow 0$ as $\varepsilon \rightarrow 0$. Indeed, if this is the case, there exists some $\delta>0$ such that $R_{j, \varepsilon} \geq \delta$ for all $j \in\{1, \ldots, N\}$, and one can easily repeat the above arguments with any of the $j$ 's in $\{1, \ldots, N\}$. Thus, in order to end the proof of the step, it remains to prove that $R_{i, \varepsilon} \not \rightarrow 0$ as $\varepsilon \rightarrow 0$ for all $i \in\{1, \ldots, N\}$. We let $i_{0} \in\{1, \ldots, N\}$ be such that, up to a subsequence,

$$
R_{i_{0}, \varepsilon}=\min _{i=1, \ldots, N} R_{i, \varepsilon}
$$

We assume by contradiction that

$$
\lim _{\varepsilon \rightarrow 0} R_{i_{0}, \varepsilon}=0 .
$$

Clearly (19) holds for $i=i_{0}$, and (36) holds. It then follows from the definition of $\mathcal{S}_{i_{0}}$ that for any $i \in \mathcal{S}_{i_{0}}$, there exists $C(i)>0$ such that

$$
R_{i, \varepsilon} \leq C(i) R_{j, \varepsilon}
$$

for all $j \in\{1, \ldots, N\}$. It follows that (19) holds for all $i \in \mathcal{S}_{i_{0}}$, and that the preceding analysis can be carried out. We pick up $i \in \mathcal{S}_{i_{0}}$ such that

$$
d_{g}\left(x_{i, \varepsilon}, x_{i_{0}, \varepsilon}\right) \geq d_{g}\left(x_{j, \varepsilon}, x_{i_{0}, \varepsilon}\right)
$$


for all $j \in \mathcal{S}_{i_{0}}$ and all $\varepsilon>0$. With (27), we get that $\left|\tilde{x}_{i_{0}}\right| \geq\left|\tilde{x}_{j}-\tilde{x}_{i_{0}}\right|$ for all $j \in \mathcal{S}_{i_{0}}$. Since $\mathcal{S}_{i}=\left(\mathcal{S}_{i_{0}} \backslash\{i\}\right) \cup\left\{i_{0}\right\}$, we have that

$$
\left|\tilde{x}_{i_{0}}\right| \geq\left|\tilde{x}_{j}-\tilde{x}_{i_{0}}\right|
$$

for all $j \in \mathcal{S}_{i}$. A consequence of this inequality is that

$$
\left(\tilde{x}_{i_{0}}, \tilde{x}_{j}\right)>0
$$

for all $j \in \mathcal{S}_{i}$, where $(\cdot, \cdot)$ denotes the Euclidean scalar product. This amounts to assuming that all the $\tilde{x}_{j}$ 's, $j \in \mathcal{S}_{i}$, lie in the same half-space where boundary contains 0 . Let $0<\delta<1$. We write thanks to equation (28) that

$$
\begin{aligned}
& \int_{B_{0}(\delta)} \nabla v_{i, \varepsilon} \Delta_{g_{i, \varepsilon}}^{2} v_{i, \varepsilon} d v_{g_{i, \varepsilon}}-r_{i, \varepsilon}^{2} \int_{B_{0}(\delta)} \nabla v_{i, \varepsilon} d i v_{g_{i, \varepsilon}}\left(A_{i, \varepsilon} \nabla v_{i, \varepsilon}\right) d v_{g_{i, \varepsilon}} \\
& =\varphi_{i, \varepsilon}\left(r_{i, \varepsilon}\right) r_{i, \varepsilon}^{4(1-\nu)} \int_{B_{0}(\delta)} f_{i, \varepsilon} \nabla e^{v_{i, \varepsilon}} d v_{g_{i, \varepsilon}}-r_{i, \varepsilon}^{4} \int_{B_{0}(\delta)} b_{i, \varepsilon} \nabla v_{i, \varepsilon} d v_{g_{i, \varepsilon}} .
\end{aligned}
$$

Integrating by parts, using the estimates of Step 2, (6) and (30), one can easily estimate the different terms involved in this equation to arrive to

$$
\int_{B_{0}(\delta)} \nabla v_{i, \varepsilon} \Delta_{g_{i, \varepsilon}}^{2} v_{i, \varepsilon} d v_{g_{i, \varepsilon}} \rightarrow 0 \text { as } \varepsilon \rightarrow 0 .
$$

Using the Cartan expansion of the metric in the exponential chart and the estimates on the derivatives of $v_{i, \varepsilon}$, some integrations by parts then lead with (30) to

$$
\begin{aligned}
\left(\int_{B_{0}(\delta)} \nabla v_{i, \varepsilon} \Delta_{g_{i, \varepsilon}}^{2} v_{i, \varepsilon} d v_{g_{i, \varepsilon}}\right)_{k} \rightarrow & -\int_{\partial B_{0}(\delta)} \partial_{k} H_{i}\left(\nabla \Delta_{\xi} H_{i}, \nu\right)_{\xi} d \sigma_{\xi} \\
& +\int_{\partial B_{0}(\delta)} \partial_{l k} H_{i} \nu^{l} \Delta_{\xi} H_{i} d \sigma_{\xi} \\
& +\frac{1}{2} \int_{\partial B_{0}(\delta)}\left(\Delta_{\xi} H_{i}\right)^{2} \nu_{k} d \sigma_{\xi}
\end{aligned}
$$

as $\varepsilon \rightarrow 0$. We let

$$
H_{i}(x)=8 \ln \frac{1}{|x|}+G_{i}(x) .
$$

Simple computations then give that

$$
\int_{B_{0}(\delta)} \nabla v_{i, \varepsilon} \Delta_{g_{i, \varepsilon}}^{2} v_{i, \varepsilon} d v_{\xi} \rightarrow 64 \pi^{2} \nabla G_{i}(0)
$$

as $\varepsilon \rightarrow 0$. Coming back to (38), we obtain that $\nabla G_{i}(0)=0$, a contradiction with the choice of $i$ we made in (37). This ends the proof of Step 3. Note that the fact that $\bar{u}_{\varepsilon} \rightarrow-\infty$ is a direct consequence of the estimate we just proved and of Step 2 .

We are now in a position to conclude the proof of Theorem 11. Using the estimates of Step 3, it is easily checked that

$$
\int_{M} f_{\varepsilon} e^{u_{\varepsilon}} d v_{g} \rightarrow 64 \pi^{2} N \text { as } \varepsilon \rightarrow 0,
$$

which gives the first assertion of the theorem thanks to (6). Since we already proved that $\bar{u}_{\varepsilon} \rightarrow-\infty$ as $\varepsilon \rightarrow 0$, it remains to prove the convergence of $u_{\varepsilon}-\bar{u}_{\varepsilon}$ outside the concentration points and to prove the last property of the theorem concerning the 
location of concentration points. We let $\mathcal{S}=\left\{x_{i}\right\}_{i=1, \ldots, N}$, where $x_{i}=\lim _{\varepsilon \rightarrow 0} x_{i, \varepsilon}$. We let $x_{0} \in M \backslash \mathcal{S}$ and we write with the Green representation formula that

$$
u_{\varepsilon}\left(x_{0}\right)-\bar{u}_{\varepsilon}=\int_{M} G_{\varepsilon}\left(x_{0}, y\right)\left(f_{\varepsilon}(y) e^{u_{\varepsilon}(y)}-b_{\varepsilon}(y)\right) d v_{g}(y),
$$

where $G_{\varepsilon}$ is the Green function of $L_{\varepsilon}$. It is then easy to compute an asymptotic expansion of the different terms involved to get that

$$
u_{\varepsilon}\left(x_{0}\right)-\bar{u}_{\varepsilon} \rightarrow 64 \pi^{2} \sum_{i=1}^{N} G\left(x_{0}, x_{i}\right)-\int_{M} G\left(x_{0}, y\right) b_{0}(y) d v_{g}(y)
$$

as $\varepsilon \rightarrow 0$, where $G$ is the Green function of the limit operator $L_{0}$. The convergence result in the theorem easily follows. The last part of the theorem is a consequence of a Pohozaev-type identity. More precisely, we write in the exponential chart around $x_{i} \in \mathcal{S}$ and for $\delta>0$ small enough that

$$
\int_{B_{x_{i}}(\delta)}\left(L_{\varepsilon} u_{\varepsilon}+b_{\varepsilon}\right) \nabla u_{\varepsilon} d v_{g}=\int_{B_{x_{i}}(\delta)} f_{\varepsilon} e^{u_{\varepsilon}} \nabla u_{\varepsilon} d v_{g}
$$

thanks to equation (4). Integration by parts together with the dominated convergence theorem then lead to

$$
\lim _{\delta \rightarrow 0} \lim _{\varepsilon \rightarrow 0} \int_{B_{x_{i}}(\delta)} f_{\varepsilon} e^{u_{\varepsilon}} \nabla u_{\varepsilon} d v_{g}=-64 \pi^{2} \frac{\nabla f_{0}\left(x_{i}\right)}{f_{0}\left(x_{i}\right)}
$$

thanks to Steps 1 to 3 and to (39). On the other hand, after integration by parts, using (39), rather long but easy computations lead to

$$
\lim _{\delta \rightarrow 0} \lim _{\varepsilon \rightarrow 0} \int_{B_{x_{i}}(\delta)}\left(L_{\varepsilon} u_{\varepsilon}+b_{\varepsilon}\right) \nabla u_{\varepsilon} d v_{g}=64 \pi^{2} \nabla G_{i}\left(x_{i}\right),
$$

where

$$
G_{i}(x)=64 \pi^{2} \beta\left(x_{i}, x\right)+64 \pi^{2} \sum_{j \neq i}^{N} G\left(x, x_{j}\right)-\int_{M} G(x, y) b_{0}(y) d v_{g}(y)
$$

with $\beta$ the regular part of $G$. The last assertion of the theorem follows.

\section{REFERENCES}

[1] Chang, S.Y.A., On a fourth-order partial differential equation in conformal geometry. Harmonic analysis and partial differential equations (Chicago, IL, 1996), 127-150, Chicago Lectures in Math., Univ. Chicago Press, Chicago, IL, 1999. MR.1743859 (2001g:58059)

[2] Chang, S.Y.A. and Yang, P.C., On a fourth order curvature invariant. Spectral problems in geometry and arithmetic (Iowa City, IA, 1997), 9-28, Contemp. Math., 237, Amer. Math. Soc., Providence, RI, 1999. MR1710786 (2001b:58056)

[3] Druet, O., From one bubble to several bubbles: the low-dimensional case, J. Diff. Geom., 63, 2003, 399-473. MR2015469 (2004h:53051)

[4] Druet, O., Compactness for the Yamabe equation in low dimensions, I.M.R.N., 23, 2004, 1143-1191. MR2041549 (2005b:53056)

[5] Druet, O. and Hebey, E., Blow-up examples for second order elliptic PDEs of critical Sobolev growth, Trans. A.M.S., 357, 2004, 1915-1929. MR2115082

[6] Druet, O., Hebey, E. and Robert, F., Blow-up theory for elliptic PDE's in Riemannian geometry, Mathematical Notes, 45, Princeton University Press, 2004. MR2063399 (2005g:53058)

[7] Kiessling, M., Statistical mechanics approach to some problems in conformal geometry, Phys. A, 279, 2000, 353-368. MR.1797146 (2003a:82003)

[8] Lin, C.S., A classification of solutions of a conformally invariant fourth order equation in $\mathbb{R}^{n}$, Comment. Math. Helv., 73, 1998, 206-231. MR1611691(99c:35062) 
[9] Paneitz, S., A quartic conformally covariant differential operator for arbitrary pseudoRiemannian manifolds. Preprint, 1983.

[10] Robert, F. and Struwe, M., Asymptotic profile for a fourth-order PDE with critical exponential growth in dimension 4, Advanced Nonlinear Studies, 4, 2004, 397-415. MR2100905

[11] Schoen, R., On the number of constant scalar curvature metrics in a conformal class. Differential geometry, 311-320, Pitman Monogr. Surveys Pure Appl. Math., 52, Longman Sci. Tech., Harlow, 1991. MR.1173050 (94e:53035)

Unité de Mathématiques Pures et Appliquées, École Normale Supérieure de Lyon, 46, allée d'Italie, 69364 Lyon cedex 7, France

E-mail address: odruet@umpa.ens-lyon.fr

Université de Nice Sophia-Antipolis, Laboratoire J. A. Dieudonné, Parc Valrose, 06108 Nice Cedex 2, France

E-mail address: frobert@math.unice.fr 